\title{
CONOCIMIENTOS, HABILIDADES Y ÉTICA PROFESIONAL DEL VALORADOR INMOBILIARIO. DIMENSIONES BÁSICAS DE UN PLAN DE FORMACIÓN
}

\author{
Ana Gómez-Bezares Revuelta ${ }^{1}$ \\ Banco de España \\ Fernando Gómez-Bezares Pascual \\ Universidad de Deusto \\ Alfredo Jiménez Eguizabal \\ Universidad de Burgos
}

RESUMEN: El presente trabajo expone los resultados de una investigación Ilevada a cabo para fundamentar una propuesta de formación habilitante para ejercer la profesión de tasador inmobiliario. Hasta este momento en España basta con ser arquitecto o ingeniero para ejercer la profesión, dejando la formación complementaria en manos de las sociedades de tasación. Mediante el análisis de los conocimientos necesarios para realizar valoraciones ajustadas, de los estándares internacionales y de apoyos empíricos, intentaremos construir un plan coherente de formación, mostrando cómo emerge la necesidad de formación en aspectos técnicos, especialmente financieros, en habilidades psicopedagógicas y la ética profesional. Con ello pretendemos reivindicar la capacidad normativa de la ciencia pedagógica como factor relevante para solucionar importantes problemas socioeconómicos, mediante el diagnóstico de necesidades formativas y el diseño de programas.

PALABRAS CLAVE: Formación, tasación inmobiliaria, habilidades psicopedagógicas, ética profesional.

\section{KNOWLEDGE, SKILLS AND PROFESSIONAL ETHICS OF REAL ESTATE APPRAISER. BASIC DIMENSIONS OF A TRAINING PLAN}

ABSTRACT: This paper presents the results of a research conducted to support a training proposal which enables students to work as real estate

1. Ana Gómez-Bezares Revuelta es inspectora de entidades de crédito del Banco de España. Este trabajo es responsabilidad exclusiva de los autores y no refleja necesariamente la opinión del Banco de España. 
appraisers. Nowadays in Spain it is enough to be an architect or an engineer to become a real estate appraiser, while further complementary training rests in the hands of the appraisal companies. Analyzing the knowledge needed to perform adjusted appraisals, the international standards and other empiric analysis, we will try to build a coherent training plan showing how the need for technical training emerges, especially in finance, in psychoeducational skills and professional ethics. Our aim is to claim the capacity of the pedagogic science as a relevant factor in solving important socio-economic problems by diagnosing training needs and the design of programs.

KEYWORDS: Education, real estate appraisal, psychoeducational skills, professional ethics.

Recibido: 11/04/2015

Aceptado: 27/07/2015

Correspondencia: Juan Alfredo Jiménez Eguizábal, Universidad de Burgos, Facultad de Educación, C/Villadiego s/n, 09001 Burgos. Email: ajea@ubu.es.

\section{INTRODUCCIÓN}

La reciente crisis iniciada en 2007 ha puesto de manifiesto, entre otros factores, errores generalizados en la tasación inmobiliaria con importantes repercusiones no solo económicas, sino también sociales y de convivencia. Durante excesivo tiempo y bajo fórmulas aparentemente neutrales, altas tasaciones difíciles de justificar y escasamente razonables, han impulsado la burbuja inmobiliaria a través de un círculo vicioso ordenado a cubrir las expectativas de tres grupos perfectamente identificados y en clara interacción: los bancos tenían interés en conceder créditos, por ello les convenía que los tasadores hicieran valoraciones elevadas que justificaran hipotecas de mayor cuantía; esta dinámica, en un entorno de muy bajos tipos de interés, llevaba a los particulares a adquirir más y más inmuebles, con la esperanza compartida por inversores, tasadores y prestamistas de que la evolución del mercado inmobiliario (continuas e importantes subidas de precios) les llevaría a los primeros a obtener plusvalías, a los segundos a hacer buenas sus altas valoraciones, y a los terceros a tener mejor garantizados sus créditos. Y el sistema funcionó correctamente hasta que el pinchazo de la burbuja inmobiliaria, junto con la crisis económica general que lo acompañó, provocó que muchos inversores no pudieran o no quisieran hacer frente a sus créditos, los bancos vieran incrementar alarmantemente sus cifras de morosidad a la vez que disminuía el valor de sus garantías, mientras las tasaciones hechas en los años previos a la crisis quedaban claramente en evidencia.

En este contexto, creemos oportuno expresar desde el principio la tesis central de este artículo que sostiene que una mejor formación de los tasadores tiene un impacto positivo en el futuro en la medida que contribuirá a que no se comentan errores. Nuestro estudio, además de tratar de mostrar la necesidad de una formación habilitante para ejercer la profesión de tasador inmobiliario, reivindica la dimensión 
pedagógica y el retorno a la formación (Santos Rego, 2013), cuyo olvido ha tenido importantes repercusiones práctico morales, afectando de forma directa a conceptos medulares del mundo educativo como los de bien común, responsabilidad o participación (Reyero, 2009).

Actualmente en España basta con tener un título de arquitecto superior o técnico -ingeniero en cierto tipo de valoraciones- para estar habilitado para ejercer la profesión de tasador, siempre que se realice este trabajo encuadrado dentro de una sociedad de tasación homologada.

Sin embargo, los conocimientos necesarios para realizar correctamente el oficio de tasador hacen que surja la necesidad de una formación más amplia y específica que la que se adquiere con una carrera de arquitectura (o de ingeniería en algunos casos). Entre otros conocimientos necesarios podríamos citar el concepto y el tratamiento del riesgo (elemento esencial en toda valoración), los bienes inmuebles y el sector inmobiliario, la valoración contable de los inmuebles, las sociedades de tasación y su sector, y los métodos de valoración que se utilizan en la tasación de inmuebles (Gómez-Bezares, 2014).

La experiencia internacional nos indica que, además, es muy importante que el tasador profesional posea determinadas habilidades y se comporte según unos estándares éticos (ATASA, 2000; IVSC, 2010, 2011, 2012; TEGoVA, 2011; AEV, 2013).

Por todo lo anterior es del máximo interés estudiar la formación que precisan los profesionales de la tasación, y, no menos importante, sus habilidades y los valores que deben impregnar su código ético. Esto último, probablemente, es una de las claves, pues existe una sospecha extendida en el sector de que ha habido influencias no deseadas que han condicionado la calidad de las tasaciones (Portillo, 2014).

Tras esta introducción el artículo se organizará de la siguiente forma: trataremos primero del acceso a la formación que proponemos, después expondremos los contenidos formativos, acabando con unas consideraciones finales a modo de conclusiones.

\section{El ACCESO A LA FORMACIÓN}

Tras analizar numerosa documentación nacional e internacional -www.atasa.es; www.asociacionaev.org; www.ivsc.org; www.tegova.org; www.rics.org-, y a la vista del mercado español de postgrados en el marco del Espacio Europeo de Educación Superior, hemos Ilegado a la conclusión de que una alternativa razonable para articular la formación de los tasadores profesionales sería el establecimiento de un "máster en valoración inmobiliaria", como título habilitante para la profesión. Para acceder al máster sería necesario poseer un grado universitario previo. La normativa española -Real Decreto 775/1997, de 30 de mayo, sobre el régimen jurídico de homologación de los servicios y sociedades de tasación- exige, para dedicarse profesionalmente a la tasación inmobiliaria homologada, la titulación previa de arquitecto o ingeniero. Tras analizar la actividad profesional de los tasadores y sus mejores prácticas, hemos llegado a la conclusión de que esta titulación previa tan específica no es necesaria, pues los conocimientos que estos profesionales necesitan se acercan más a los de un economista. En general, cualquier carrera de ciencias donde se adquieran unos conocimientos en matemáticas y estadística podría ser suficiente para acceder a esta formación. 
En efecto, los aspirantes a este curso deberían ser competentes en:

$1^{\text {a }}$ competencia previa: utiliza las matemáticas al nivel de manejar con soltura ecuaciones de grado $n$ y el tipo de interés simple y compuesto para realizar valoraciones aplicando los métodos habituales de descuento de flujos.

$2^{a}$ competencia previa: maneja la estadística al nivel de utilizar con agilidad las medidas descriptivas de posición y dispersión, comprender los elementos básicos de estadística inferencial e interpretar regresiones lineales y no lineales para realizar valoraciones aplicando los métodos utilizados en el sector y los descritos en la normativa española -Orden ECO/805/2003, de 27 de marzo, sobre normas de valoración de bienes inmuebles y de determinados derechos para ciertas finalidades financieras-.

$3^{\text {a }}$ competencia previa: conoce la informática a nivel de usuario en un entorno Windows y emplea el ordenador manejando office y en especial la hoja de cálculo a un nivel básico, así como efectúa con agilidad búsquedas en internet, para realizar valoraciones aplicando los métodos utilizados en el sector y los descritos en la normativa española.

A la vista de lo anterior creemos que un alumno que ha superado el bachiller habiendo cursado matemáticas al nivel previamente expuesto, debería poseer estas tres competencias previas. Aceptado esto, cualquier graduado universitario debería poder acceder a la formación que estamos proponiendo: podemos entender que el grado universitario le proporcionaría, entre otras cosas, las competencias genéricas y el grado de madurez necesario, mientras las competencias específicas previas habrían sido adquiridas con anterioridad.

En todo caso, una alternativa podría ser establecer un proceso de selección para la entrada en el máster, que incluya una prueba en la que el aspirante pueda demostrar su destreza en las tres competencias previas. Seríamos partidarios de hacer esa prueba obligatoria para todos los estudiantes que no hayan tenido asignaturas de matemáticas y estadística en su programa de grado. También se podría establecer una preferencia por los graduados en carreras de economía, arquitectura o ingeniería; y esto último basado no solo en los conocimientos, sino también en las previsibles actitudes y motivaciones de los aspirantes.

\section{Contenidos formativos}

En este apartado vamos a analizar los contenidos mínimos de formación que debe poseer el profesional de la valoración inmobiliaria. Seguiremos de una manera especial los "Minimum Educational Requirements" (en adelante, MER) desarrollados por TEGoVA (2011) así como IVSC (2010, 2012, 2013). Dado su impacto en el sector, fundamentalmente a nivel europeo, Gómez-Bezares (2014) sigue la conceptualización y nomenclatura propuesta por TEGoVA que habla de "Syllabus", "Purposes" y "Objectives", que traduciremos como Syllabus (plan), Propósitos y Objetivos.

TEGoVA (2011) reconoce entre sus fines que "seeks to ensure high standars of profesional competence in valutation", para lo que TEGoVA requiere a sus asociados que fijen estándares educacionales al menos tan exigentes como los MER. Los MER fueron introducidos en 2003 y han sido actualizados posteriormente, y siguen la Directiva 1992/51/CE relativa al reconocimiento mutuo de las cualificaciones profesionales. También reconoce 
TEGoVA que la industria no está solo orientada a la valoración en las transacciones, sino que los valoradores son consultados para diferentes actividades corporativas que pueden ir desde la consultoría estratégica hasta la responsabilidad social corporativa.

En este artículo nos vamos a centrar en el esquema general del Syllabus sin entrar en su desarrollo con los purposes y objetives (que pueden verse en GómezBezares, 2014). Tales purposes pueden aproximarse a las "competencias" y los objetives a los "resultados del aprendizaje" (Gómez-Bezares, 2014: 408, nota 118). Lo que sí haremos es detenernos un poco más en "las finanzas", al ser el núcleo de la formación del valorador, proponiendo un programa general de las dos materias de finanzas; expondremos una relación no exhaustiva de habilidades y nos detendremos en la dimensión ética por su transcendencia a nivel personal y social, más tras una crisis que ha estado claramente influenciada por la falta de valores éticos.

\section{Syllabus: Esquema general}

Siguiendo el documento de TEGoVA (2011) dividiremos el Syllabus en tres niveles:

- Nivel de comprensión

- Nivel de conocimiento general

- Nivel de conocimiento en profundidad

En base a los documentos antes citados, y a nuestra propia experiencia profesional, las materias que se deberían incluir en cada nivel serían las que se pueden encontrar en la figura 1.

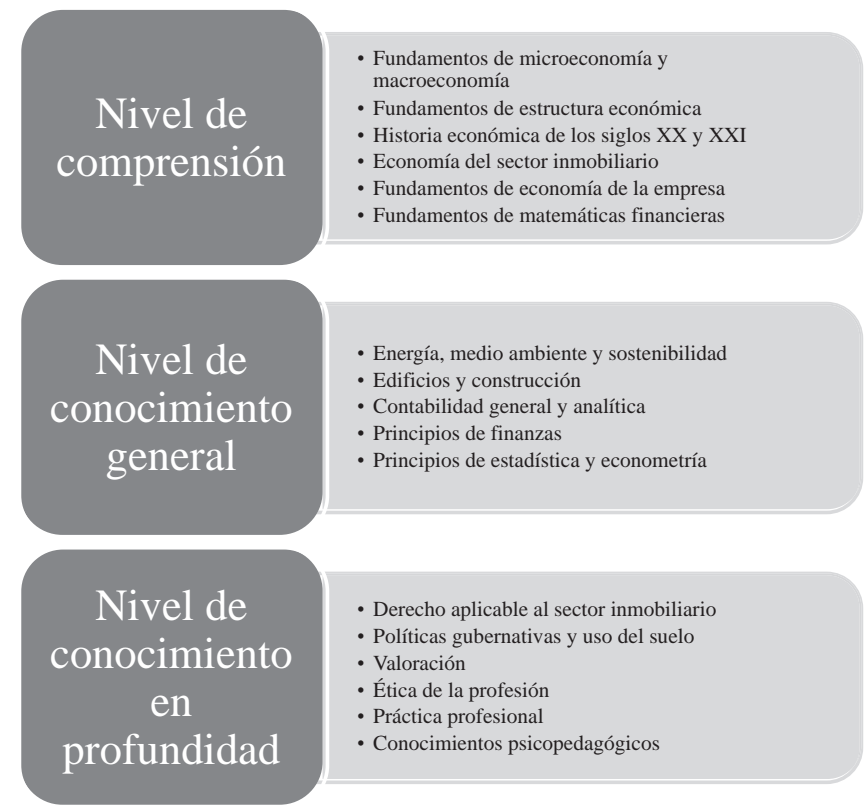

Figura 1. Plan de Formación Fuente: elaboración propia 


\section{Las finanzas como núcleo medular en la formación del valorador}

Las finanzas se ocupan de la valoración y el riesgo (Mascareñas, 2013). En consecuencia, toda valoración en condiciones de riesgo, incluida por lo tanto la valoración inmobiliaria, es objeto de atención por la ciencia financiera. Las finanzas, por tanto, deben aportarnos el núcleo de la formación del tasador, a pesar de que esto no ha sido hasta ahora explícitamente contemplado por la legislación española. La realidad es que cualquier análisis que hagamos sobre la orden ECO/805/2003, base de nuestro sistema de valoración inmobiliaria para fines hipotecarios, o de los sistemas de valoración utilizados por los profesionales del sector en cualquier parte del mundo, nos Ileva a que la valoración tiene un fundamento claramente financiero.

En efecto, las finanzas nos proporcionan los argumentos fundamentales para la valoración: el valor del dinero en el tiempo, el riesgo, la financiación, la valoración contable, los métodos de valoración... Cualquier manual de finanzas (Brealey, Myers y Allen, 2010; Brealey, Myers y Marcus, 2007; Gómez-Bezares, 2012) puede corroborar estas ideas.

Por otro lado la prestigiosa revista internacional especializada en educación y sector inmobiliario: Journal of real estate practice and education, que obviamente ha sido una de las fuentes principales en este trabajo, reconoce en varios artículos la importancia central de las finanzas en la formación del valorador. Así, en una relevante contribución, los investigadores Tu, Weinstein, Worzala y Lukens (2010) en base a una encuesta realizada a stakeholders del sector inmobiliario concluyen que las finanzas son consideradas claramente el tema más crítico en el curriculum del graduado en el sector inmobiliario, los cursos de finanzas fueron señalados como los más importantes con un amplio margen y la modelización financiera con Excel fue la práctica más valorada por todos los grupos de stakeholders.

Además las grandes asociaciones profesionales -The European Group of Valuers' Associations (TEGoVA), International Valuation Standards Council (IVSC), Royal Institution of Chartered Surveyors (RICS), Asociación Española de Análisis de Valor (AEV), entre otras- reiteran la importancia central de las finanzas en la formación del tasador (TEGoVA, 2011, 2012; IVSC, 2010, 2011, 2012, 2013; RICS, 2008; y AEV, 2013).

Por último, la supervisión de las sociedades de tasación homologadas corresponde, según la legislación española, al Banco de España. Es claro que el legislador español -Ley 3/1994, de 14 de abril, por la que se adapta la legislación española en materia de entidades de crédito a la segunda directiva de coordinación bancaria y se introducen otras modificaciones relativas al sistema financiero- entendió que esa supervisión debía realizarse desde un supervisor financiero como es el Banco de España.

A la vista de todo lo anterior pasamos a enunciar (figura 2) los temas principales del programa de finanzas, que respondería a la materia "principios de finanzas" en los temas 1 al 6 y "valoración" de los temas 7 al 12.

Este programa puede apoyarse en Aragón et al. (2010), Brealey, Myers y Allen (2010), Brealey, Myers y Marcus (2007), Copeland, Koller y Murrin (2000), Cuervo, Rodríguez, Calvo y Parejo (2012), García Almirall (2007), Gómez-Bezares (2012), Gómez-Bezares, Madariaga, Santibáñez y Apraiz (2013), González, Turmo y Villaronga (2006), Guadalajara (2014), Martín Marín y Trujillo (2004); así como en los 
estándares de TEGoVA (2012), RICS (2014), IVSC (2013), AEV (2013), ATASA (2000 y 2011); la Orden ECO/805/2003, las Normas Internacionales de Contabilidad (NICs) y las Normas Internacionales de Información Financiera (NIIFs).

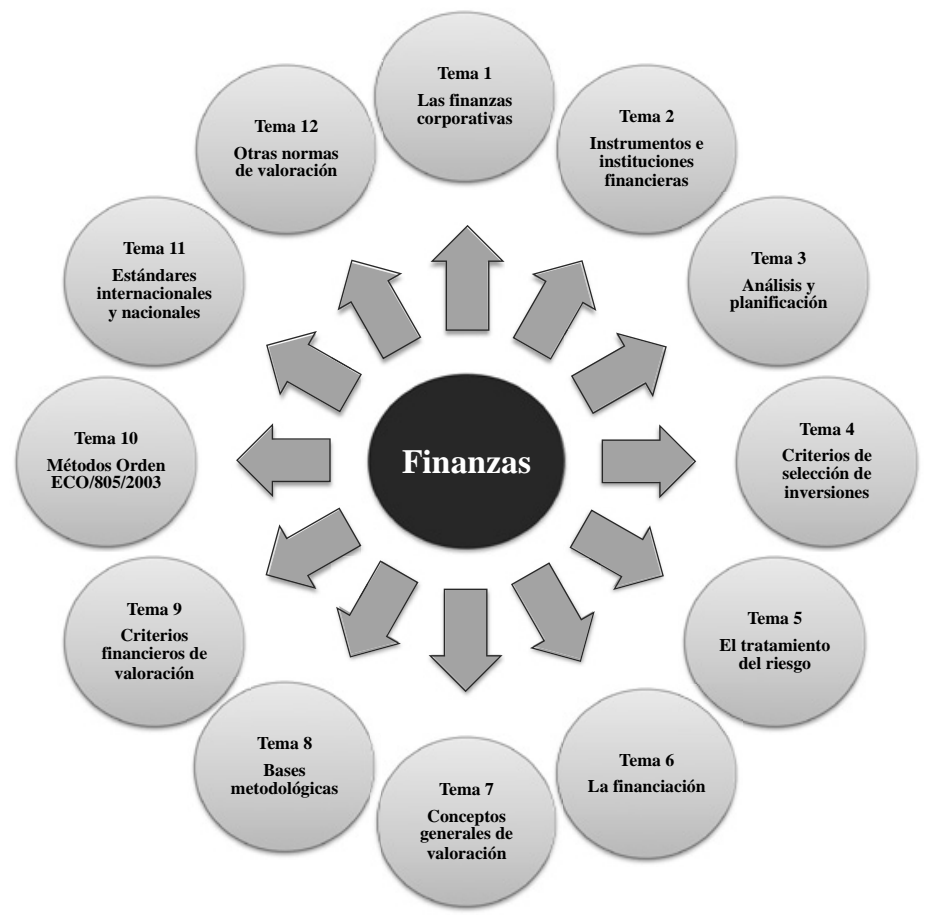

Figura 2. Programa de Finanzas. Fuente: Elaboración propia

\section{Habilidades necesarias}

Las asociaciones profesionales suelen añadir a las competencias específicas que debe tener el valorador -y que deberá adquirir con las materias anteriormente enumeradas- unas skills o habilidades que coinciden básicamente con lo que solemos denominar "competencias genéricas". Se entiende que tales competencias genéricas han sido ya adquiridas por el futuro valorador en el grado previo que le daría acceso a la formación específica que venimos señalando; con todo parecería razonable que esas competencias genéricas también se desarrollasen en la formación citada, lo que haríamos en las diferentes materias y de manera especial en la materia "Conocimientos psicopedagógicos". Siguiendo a IVSC (2010), que emite estándares habituales en la profesión, plantearemos las habilidades que parecen más necesarias en un valorador profesional:

- Habilidades para la resolución de problemas, analíticas y de investigación

- Donde se incluye el pensamiento creativo para generar soluciones innovadoras

- Y la capacidad de organizar y comunicar informes técnicos por medios escritos, electrónicos y orales 
- Habilidades interpersonales

- Donde se incluye la capacidad de trabajo en equipo

- Y las capacidades de liderazgo, motivación y empoderamiento de equipos e individuos

- Habilidades de comunicación y escucha

- Donde se incluye la capacidad de persuasión oral y escrita

- Habilidades tecnológicas

- Que enfatiza el dominio de las tecnologías actuales y emergentes en materia de información y comunicación: TICs, ofimática, etc.

- Y su uso en las operaciones empresariales y en la toma de decisiones

- Habilidades para la gestión de proyectos

- Donde se incluye desde la capacidad de reacción ante problemas inesperados o la capacidad de priorización y gestión del tiempo, hasta la conciliación de la vida profesional y familiar

- La orientación a la calidad y la orientación al logro

- Y la capacidad para crear amplios equipos interdisciplinares cuando sea preciso

- Habilidades para emitir juicios equilibrados

- Donde se incluye la capacidad para llegar a conclusiones bien razonadas en base a los hechos relevantes, así como para mantener una actitud crítica respecto a las soluciones establecidas

- Y obtener información de calidad, manejarla correctamente y ser objetivos para llegar a la conclusión apropiada

Como indica IVSC (2010), que ha inspirado esta lista de habilidades, esta relación incluye algunas de las más características, pero no pretende ser exhaustiva.

Por otro lado y previamente a la formación propuesta, simultáneamente con ella, o como complemento final, deberán incluirse en la misma:

- Nivel de inglés. Dado el nivel de globalización de la actividad económica, y de forma especial de la actividad financiera a la que la tasación está íntimamente ligada, parece imprescindible un cierto nivel de inglés en estos profesionales. Por poner solo dos ejemplos: todos los estándares a los que nos hemos venido refiriendo, excepto los españoles, están en inglés; y la nueva supervisión única del Banco Central Europeo, cuyo idioma habitual es el inglés, sobre las entidades españolas está impulsando el uso del inglés en el sector.

- Experiencia. Es una opinión unánime en el sector de la tasación que la experiencia es un activo fundamental para un tasador. Conocer la operativa profesional, y sobre todo el mercado inmobiliario concreto, es el mejor aval para realizar una buena tasación. Ese "olfato" para presumir el precio de un bien inmobiliario, y sobre todo para descartar valoraciones desenfocadas, solo se adquiere con la experiencia, muchas veces apoyada por el sentido común. Evidentemente los métodos de valoración son un apoyo importante, pero sin ese "olfato" estaríamos demasiado expuestos al error. Se puede hacer el símil del inversor bursátil: los descuentos de flujos, los multiplicadores, el análisis 
técnico... le serán sin duda útiles, pero siempre hay inversores que destacan sobre los demás por su buen olfato. Por todo lo anterior es evidente que el aspirante a tasador debería, antes de volar solo, adquirir una buena experiencia en el sector concreto en el que va a desarrollar su actividad.

- Prácticas. A nivel de conocimiento en profundidad, hablamos de la materia "Práctica profesional". Dada su ubicación en el Syllabus es claro que hemos querido darle importancia, y se considera imprescindible, como complemento a esta materia, el que los estudiantes hicieran prácticas en alguna tasadora homologada mediana o grande. Estas prácticas podrían ser convalidadas si se acredita una suficiente experiencia previa.

\section{La dimensión ética}

La formación ética ya la hemos señalado en el Syllabus en la materia de "Ética de la profesión". Allí aparece a un nivel de conocimiento en profundidad, lo que indica la importancia capital que damos a esta materia. Con todo, el problema es que la ética, sobre todo, debe ser practicada: el introducirla en un programa de formación puede ayudar a sensibilizar al futuro profesional, puede incluso alertarle de las graves consecuencias que los comportamientos poco éticos pueden conllevar, pero será el individuo en uso de su libertad el que al final decida su comportamiento. Para tranquilizar a la sociedad podemos recordar que existen las leyes y los códigos profesionales (Appraisal Institute, 2013), con sus correspondientes sistemas de vigilancia y sanción, de manera que esa libertad del individuo quede limitada. Pero al final siempre hay un margen de maniobra dentro del cual el individuo es responsable ante su conciencia.

Los problemas éticos de la profesión de tasador pueden encuadrarse sin mucha dificultad entre los problemas éticos de las profesiones financieras. Así, creemos que los problemas más característicos de la profesión son el uso indebido de la información y los conflictos de interés (Ansotegui, Gómez-Bezares y González Fabre, 2014; Escámez, 2003).

En efecto, el tasador maneja información que es confidencial, como puede ser el caso de que tenga que tasar un inmueble porque su propietario ha pasado a la cartera de dudosos de la entidad bancaria: esta situación financiera de dicho propietario no es una información pública; también se puede pedir una tasación previa a un divorcio o en el proceso de reparto de una herencia, y que el cliente no quiera hacer pública dicha petición. Por otro lado, puede ocurrir que el tasador tenga posibilidad de acceder a información privilegiada, que si llegara a sus manos, no debería usar: pensemos en una vivienda con un valor objetivo de $500 \mathrm{~m} €$, su propietario ha recibido una oferta de un importante despacho de abogados instalado en el piso contiguo, y solicita una tasación antes de plantearse el atender o no a dicha oferta, el tasador ha logrado enterarse, sobornando a la secretaria del despacho, de que los abogados están dispuestos a subir dicha oferta hasta los $2 \mathrm{M} €$; es evidente que el tasador no actuaría éticamente utilizando esa información en su tasación. Tampoco sería ético el que usara esa información si le hubiera llegado porque dos de los socios del despacho estaban comentándolo en una mesa cercana a la suya en un bar.

Es probable que todavía sean más frecuentes los conflictos de interés. El tasador debería actuar como un profesional independiente, pero, desgraciadamente, puede 
ser objeto de diferentes presiones que acaben alterando su objetividad de juicio. Presiones que le pueden Ilegar desde la entidad financiera de la cual depende una amplia proporción de la facturación de su sociedad de tasación, de la propia sociedad de tasación de la que el tasador es simplemente un colaborador o del cliente que le pide la tasación de un inmueble y lo presiona de variadas maneras. La falta de independencia de las sociedades de tasación y de sus tasadores puede ser la causa de muchas tasaciones incorrectas. Pero sin llegar a hacer tasaciones erróneas, en el mundo de la valoración siempre hay margen para la opinión, por lo que la falta de independencia puede ser un problema grave. Nuestra experiencia nos indica que muchos agentes del sector desearían que la independencia de los tasadores estuviera más salvaguardada, y la legislación, los códigos profesionales y la ética de los agentes deberían ir avanzando en preservarla de una manera más eficaz.

Un conflicto de interés relacionado con el uso de la información es la debida transparencia con las administraciones, con los clientes, con la sociedad. En muchas ocasiones la sociedad de tasación debería dar una información que no le conviene dar. Por otro lado la transparencia puede ser una garantía de una actuación más ética: es más difícil hacer las cosas mal si sabes que todo el mundo se va a enterar.

Otros temas que podríamos discutir respecto a la actuación ética de los valoradores serían la posibilidad de manipular precios, los problemas de agencia o la gestión del tiempo. Respecto al primero no tenemos evidencias de que haya sido un problema importante, lo que no impide estar vigilantes sobre ello; de todas formas podríamos interpretar la burbuja inmobiliaria que explota en 2008 como una manipulación de precios, si bien aquí la responsabilidad de los tasadores la compartieron con banqueros, autoridades gubernativas y público en general.

El problema de agencia, entendido en este caso como que el tasador no actúa como un agente leal de los intereses de su sociedad de tasación y de los partícipes sociales o stakeholders en general, sino por su propio interés particular, se da en el mundo de la valoración inmobiliaria igual que ocurre en la banca, en los seguros o en la mayoría de las actividades económicas.

Por último pueden ser importantes en esta profesión los problemas de gestión del tiempo, que se pueden presentar cuando un tasador prioriza y se esmera más en una tasación más lucrativa bajando mucho sus exigencias de calidad en otra; o las limitaciones que la vida laboral pueda imponer a la familiar.

Podríamos sin duda identificar otros dilemas éticos en la profesión del tasador, aunque creemos que hemos relatado los más característicos. Nuestra conclusión aquí sería que la independencia resulta fundamental, por lo que desde los tasadores hasta el supervisor deberían esforzarse en preservarla. Asumiendo que el tasador posee unos valores éticos, el que actúe con independencia va a obedecer en muchas ocasiones a su independencia económica, lo que a su vez tendrá mucho que ver con sus alternativas laborales y con su endeudamiento: "la fidelidad a tu empresa depende del tamaño de tu hipoteca". Sin duda un mercado laboral que ofrezca alternativas a los tasadores ayudará a que sea más fácil que mantengan su independencia. Sin embargo esto último no depende del tasador; lo que sí depende de él es no llevar un ritmo de gasto o inversión que le lleve a un nivel de endeudamiento que limite totalmente su independencia. 


\section{CONSIDERACIONES FINALES}

Partiendo de que unas tasaciones ajustadas nos ayudarán en el futuro a evitar otra burbuja inmobiliaria, aunque sin pretender poner bajo sospecha a los profesionales, ni que las tasaciones hayan sido las únicas responsables de lo que ha ocurrido, este trabajo ha indagado en cómo capacitar al tasador para que realice correctamente su trabajo. A través de los referentes empíricos utilizados hemos ido verificando los supuestos planteados en las consideraciones iniciales, esto es, la necesidad de abordar una formación habilitante para ejercer la profesión de tasador inmobiliario, evidenciando la sustantiva relación entre formación correcta, desarrollo económico y el compromiso social sujeto a principios éticos (Santos Rego, 2009).

El método de investigación en este trabajo se ha fundamentado en una amplia revisión documental, sobre todo de los estándares internacionales sobre la formación de tasadores, así como de los nacionales. Pero también en revisiones bibliográficas, de prensa, documentación utilizada y publicada por las sociedades de tasación y por las asociaciones del sector, etc. (Gómez-Bezares, 2014). Todo esto se ha hecho además partiendo de los conocimientos profesionales de los autores en los campos de interés de este trabajo: la educación y la valoración inmobiliaria. En todo caso esta investigación tiene limitaciones al haberse hecho, sobre todo, desde el punto de vista de lo que es y necesita el tasador inmobiliario para ejercer su profesión y podría complementarse con un análisis de la actual oferta formativa a nivel nacional e internacional. Evidentemente este análisis lo hemos hecho, pero no lo hemos explicitado dado que nuestro énfasis lo hemos puesto en lo que el sector entiende como necesario. Incluso esta perspectiva se podría ampliar con otras metodologías como la encuesta, el análisis de datos, etc. Pero dejamos estas líneas para futuras investigaciones. Con todo, sí hemos constatado que los programas formativos existentes en España no tienen el sesgo financiero que nosotros defendemos como característico en la formación de cualquier "valorador", y que propugnan las más recientes demandas internacionales. Por otro lado defendemos que esa formación sea habilitante para el ejercicio profesional, cosa que funciona diferente en nuestro país.

Para constatar la amplia variedad de regulación para el ejercicio de la profesión de valorador inmobiliario, baste como ejemplo la heterogeneidad reinante en Europa, como puede comprobarse en Gómez-Bezares, Gómez-Bezares y Jiménez (2015).

El destinatario de esta formación es el futuro tasador inmobiliario. Si además conseguimos que la regulación considere esta formación como habilitante, siguiendo en este caso la tradición anglosajona, todos los futuros tasadores deberán contar con esta formación. Tal y como hemos dicho, la formación diseñada proponemos que se articule como un máster universitario con sus correspondientes requisitos de acceso, si bien podría tener otros formatos, incluso como título propio.

A la vista de los conocimientos necesarios para la valoración inmobiliaria, nos parece poco justificable que con una titulación de arquitectura o ingeniería, sin ningún complemento, pueda ejercerse directamente la profesión de tasador. No hay duda de que la formación que obtiene un arquitecto o un ingeniero puede ayudarle a entender mejor algunos aspectos de la valoración del inmueble, pero lo que es la valoración en sí está más en la línea de la formación de un economista. Por todo ello, una buena solución podría ser que graduados en alguno de estos tres campos pudieran acudir a 
la formación propuesta. Tal formación pretende ser bastante completa, en consonancia con los estándares internacionales, por lo que en función de la formación previa resultarán más novedosas unas áreas de conocimiento, destacando la prevalencia de las finanzas como ciencia de la valoración, que otras. A este respecto, también hemos tratado de hacer una relación, no exhaustiva, de competencias genéricas de acuerdo con los estándares internacionales de IVSC.

En esta línea, hemos intentado mostrar cómo inciden en el plan de formación un importante conjunto de habilidades psicopedagógicas que aportan sensibilidad, capacidad innovadora, practicidad y funcionalidad.

Por último, pero con evidente influencia y repercusión, hay que ponderar la capacidad formativa de la ética, que viene justamente a coincidir con las nuevas demandas sociales. Junto a la estimación de los conocimientos técnicos y habilidades psicopedagógicas aparece la dimensión ética que proporciona al profesional argumentos y criterios para responder con acierto, en medio de la incertidumbre de un sistema económico y social en cambio, al interrogante kantiano en torno a cómo debo comportarme.

El que el tasador sea independiente no garantiza su habilidad técnica, tampoco puede asegurar su honestidad, simplemente posibilita el que pueda utilizar correctamente sus habilidades técnicas y hacer honestamente su trabajo. A contrario sensu: si el tasador carece de independencia se convertirá en un "portavoz" de intereses ajenos, muchas veces enfrentados con su deber profesional, y de poco servirán sus competencias técnicas. Independencia, prudencia y justicia serán buenas compañeras en el camino profesional del valorador inmobiliario, como lo son en las profesiones relacionadas con el mundo financiero.

La propuesta es viable y sostenible, pues dada la importancia del sector tasador en todos los países desarrollados, la formación de sus profesionales es un proyecto de interés social que no puede ser desatendido por los poderes públicos y tendrá, sin duda, también interés para los agentes privados; si bien no es objeto de este artículo detallar una memoria económica sobre este proyecto formativo.

En una sociedad sujeta a las incertidumbres de la complejidad y a los retos de un mundo globalizado, resulta relevante constatar que en los procesos de toma de decisiones en torno a las cuestiones más disputadas, además de la efectividad, siguen importando los valores y el sentido normativo-crítico que aporta el proceso intencional y perfectivo sustantivo de la formación (Jiménez, Palmero y Jiménez, 2012). Y con ello, también se amplía la racionalidad instrumental, crítica y creativa de la Pedagogía, así como su capacidad normativa para diseñar y coordinar planes de formación.

\section{REFERENCIAS BIBLIOGRÁFICAS}

AEV (2013). Estándares profesionales de los miembros de la Asociación Española de análisis del Valor (AEV) sobre medios técnicos y humanos de los que deben disponer las sociedades de tasación para abordar las actividades de valoración. Recuperado de http://cort.as/WpZQ.

Ansótegui, C., Gómez-Bezares, F., y González Fabre, R. (2014). Ética de las finanzas. Bilbao: Desclée de Brouwer. 
Appraisal Institute (2013). Code of professional ethics of the Appraisal Institute Recuperado de http://www.appraisalinstitute.org/assets/1/7/CPE.pdf.

Aragón, R., Gámez, S., Gómez-Bezares, F., Lamothe, P., Mascareñas, J., y Viñolas, P. (2010). La valoración de la empresa ( $2^{\mathrm{a}}$ ed.). Madrid: Consejo General de Colegios de Economistas de España.

Atasa (2000). Código ético de la Asociación Profesional de Sociedades de Valoración. Atasa (2011). Estándar de medición de superficies de inmuebles.

Brealey, R. A., Myers, S. C., y Allen F. (2010). Principios de finanzas corporativas. México D. F.: McGraw-Hill.

Brealey, R. A., Myers, S. C., y Marcus, A. J. (2007). Fundamentos de finanzas corporativas. Madrid: McGraw-Hill.

Copeland, T., Koller, T., y Murrin, J. (2000). Valuation, measuring and managing the value of companies. Nueva York: Wiley.

Cuervo, A., Rodríguez, L., Calvo, A., y Parejo, J. A. (2012). Manual del sistema financiero español. Barcelona: Ariel.

Directiva 1992/51/CEE del Consejo, de 18 de junio de 1992, relativa a un segundo sistema general de reconocimiento de formaciones profesionales, que completa la Directiva 89/48/CEE (DOL, 24 de julio de 1992, nº 209).

Escámez, J. (2003). Pensar y hacer hoy educación moral. Teoría de la Educación, 15, 21-31.

García Almirall, M. P. (2007). Introducción a la valoración inmobiliaria. Barcelona: Departament de Construccions Arquitectòniques I Escola Tècnica Superior d'Arquitectura de Barcelona Càtedra d'Arquitectura Legal Dret Urbanístic i Valoracions.

Gómez-Bezares, A. (2014). Valoración inmobiliaria profesional: método y aptitudes (Tesis Doctoral, Universidad de Burgos).

Gómez-Bezares, F. (2012). Elementos de finanzas corporativas. Bilbao: Desclée de Brouwer.

Gómez-Bezares, F., Gómez-Bezares, A., y Jiménez, A. (2015). Regulación de la tasación inmobiliaria. Revista de derecho, empresa y sociedad, 6, 155-172.

Gómez-Bezares, F., Madariaga, J. A., Santibáñez, J., y Apraiz, A. (2013). Finanzas de empresa (selección de lecturas). Bilbao: Deusto Business School. Recuperado de http://www.eumed.net/libros-gratis/2013a/1290/.

González, P., Turmo, J., y Villaronga, E. (2006). La valoración inmobiliaria. Madrid: La Ley.

Guadalajara, N. (2014). Métodos de valoración inmobiliaria. Madrid: Mundiprensa.

IVSC (2010). Definition of a Professional Valuer. Londres: International Valuation Standards Council.

IVSC (2011). Code of Ethical Principles for Professional Valuers. Londres: International Valuation Council.

IVSC (2012). A Competency Framework for Professional Valuers. Londres: International Valuation Standards Council.

IVSC (2013). International Valuation Standards 2013: Framework and Requirementes. Londres: International Valuation Standards Council. 
Jiménez, A., Palmero, C., y Jiménez, J. A. (2012). El impacto de la educación secundaria y superior en la creación de empresas en la Unión Europea. Revista Española de Pedagogía, 252, 201-219.

Ley 3/1994, de 14 de abril, por la que se adapta la legislación española en materia de entidades de crédito a la segunda directiva de coordinación bancaria y se introducen otras modificaciones relativas al sistema financiero $(B O E, 15$ de abril de 1994, n 90).

Martín Marín, J. L., y Trujillo, A. (2004). Manual de Mercados Financieros. Madrid: Paraninfo.

Mascareñas, J. (2013). Introducción a las finanzas corporativas. Monografías de Juan Mascareñas sobre Finanzas Corporativas. Recuperado de http://ssrn.com/ abstract $=2313264$.

NICs. Normas Internacionales de Contabilidad. International Accounting Standards Board (IASB). Recuperado de http://cort.as/3oUZ.

NIIFs. Normas Internacionales de Información Financiera. International Accounting Standards Board (IASB). Recuperado de http://cort.as/3oUZ.

Orden ECO/805/2003, de 27 de marzo, sobre normas de valoración de bienes inmuebles y de determinados derechos para ciertas finalidades financieras $(B O E, 9$ de abril de 2003, n 85).

Portillo, J. (2014). La banca pasa de tener una docena a sólo tres tasadoras en la crisis, Cinco Días, Noticia del 22 de septiembre de 2014. Recuperado de http://cort.as/ WpdW.

Real Decreto 775/1997, de 30 de mayo, sobre régimen jurídico de homologación de los servicios y sociedades de tasación (BOE, 13 de junio de 1997, n 141).

Reyero, D. (2009). El peso del Estado en la educación. Una aproximación crítica desde la economía liberal. Revista Española de Pedagogía, 244, 409-425.

RICS (2008). Estándares RICS de valoración: versión en castellano. Coventry: RICS Books Surveyor Court.

RICS (2014). RICS Valuation - Professional Standards. Coventry: RICS Books, Surveyor Court.

Santos Rego, M. A. (Coord.) (2009). Políticas educativas y compromiso social. Madrid: Octaedro, Ministerio de Educación.

Santos Rego, M. A. (2013). Cosmopolitismo y educación. Aprender y trabajar en un mundo sin fronteras. Valencia: Brief.

TEGoVA (2011). Minimun Educational Requirements (MER), The European Group of Valuers' Associations. Recuperado de http://www.tegova.org/en/p4912f882b2055.

TEGoVA (2012). European Valuation Standards. The European Group of Valuers' Associations, Bélgica.

Tu, C. C., Weinstein, M., Worzala, E., y Lukens, L. (2010). Elements of Successful Graduate Real Estate Programs: Perceptions of the Stakeholders. Journal of Real Estate Practice and Education, 12(2), 105-121. 